\title{
Opportunities to improve recruitment into medical genetics residency programs: survey results of program directors and medical genetics residents
}

\author{
Michelle Cichon, $\mathrm{MS}^{1}$ and Gerald L. Feldman, $\mathrm{MD}, \mathrm{PhD}^{1-3}$
}

\begin{abstract}
Purpose: Approximately 50\% of medical genetics residency positions remain unfilled each year. This study was designed to assess current recruitment strategies used by program directors, to identify factors that influenced trainees to choose medical genetics as a career, and to use these results as a foundation to develop a strategic plan to address the challenges of recruitment.
\end{abstract}

Methods: Two surveys were created, one for program directors and one for current medical genetics residents, to evaluate current recruiting efforts and institutional support for programs and to identify factors that helped trainees choose genetics as a career.

Results: Program directors identified the most successful recruiting methods as "direct contact with residents or medical students" and "word of mouth" (80\%). Residents listed having a mentor (50\%), previous research in genetics (35\%), and genetics coursework (33\%) as the top reasons that influenced them to enter the field.

Conclusion: Geneticists should become more proactive in providing resources to students to help them understand a career as a medical geneticist and mentor those students/residents who show true interest in the field. Results of these surveys spurred the development of the Task Force on Medical Genetics Education and Training of the American College of Medical Genetics and Genomics.

Genet Med advance online publication 17 October 2013

Key Words: medical genetics education; medical genetics residency; medical genetics residents; program directors; recruitment

\section{INTRODUCTION}

Advances in genetic technology and discovery have provided better diagnostic tools and more treatment options for genetic disease; however, the number of trainees choosing the primary specialty of medical genetics is not increasing. As more common diseases are shown to have a genetic component, there is evidence that an inadequate number of medical geneticists are being trained to take care of the needs of those affected with all genetic diseases. ${ }^{1}$ Various medical care models have been proposed to care for patients with common diseases, including integration into primary care and a collaboration of geneticists and other professionals (e.g., cardiologists, neurologists). ${ }^{2}$ Even with these changes, the need for clinical geneticists will still exist through any transition of care because primary care physicians do not feel adequately prepared to assess family histories for hereditary conditions. ${ }^{3}$

Statistics reveal the low number of board-certified medical geneticists. As of the 2011 American Board of Medical Genetics (ABMG) certification cycle, the number of certified Doctor of Medicine/Doctor of Osteopathic Medicine (MD/DO) clinical geneticists was just $>1,400 .{ }^{4}$ More than a third were certified in the 1980s, resulting in many approaching retirement age. In February 2012, there were 115 clinical genetics trainees who took the genetics in-service examination (N. Robin, unpublished data) compared with 127 trainees the previous year. ${ }^{5}$ Although perhaps not a significant difference, it demonstrates that the numbers are not increasing. Forty-five residents completed their categorical medical genetics training in 2012. ${ }^{6}$ Data for the combined programs were not available from the Accreditation Council for Graduate Medical Education, which would add a few additional graduates each year. Furthermore, the National Residency Matching Program reported that there were 52 certified categorical medical genetics residency slots in the Specialties Match ${ }^{\mathrm{SM}}$ for the 2012 appointment year, of which only 29 (56\%) were filled. ${ }^{7}$ The 2013 appointment year revealed 53 available positions, again with only 25 (47\%) being filled. ${ }^{8}$ The 2013 Main Residency Match $^{\mathrm{SM}}$, which includes combined programs and the 4-year categorical program, had 12 available positions, with only 7 being filled (58\%). However, there were originally 12 pediatrics-medical genetics programs participating and only 9 slots were offered, which means that some programs withdrew their available positions before the Match. ${ }^{9}$ These statistics demonstrate the lack of trainees entering the field, the lack of filling of all offered positions, and the lack of offering of all available positions.

In October 2004, the first Banbury Summit meeting on training of physicians in medical genetics was held. The goal was to increase the number of residents who receive training in medical genetics. The Banbury Report suggested the need for data

${ }^{1}$ Center for Molecular Medicine and Genetics (CMMG), Wayne State University School of Medicine, Detroit, Michigan, USA; ${ }^{2}$ Department of Pathology, Wayne State University School of Medicine, Detroit, Michigan, USA; ${ }^{3}$ Department of Pediatrics, Wayne State University School of Medicine, Detroit, Michigan, USA. Correspondence: Michelle Cichon (mcichon@med.wayne.edu) 
collection, such as determining the perceptions of medical students about medical genetics as a career. ${ }^{10}$ One goal of this study was to obtain such data by surveying current medical genetics residents' reasons for choosing medical genetics. The objectives of this study were to (i) identify the demographics of those drawn to the field, (ii) determine the reasons for which one chooses medical genetics, (iii) determine when that career decision is made, (iv) define what factors influence that decision, and (v) use the results as a foundation to develop a strategic plan to address the challenges facing this specialty. In a second survey, medical genetics residency program directors were surveyed to assess current recruitment strategies to identify successful approaches and challenges that prevent programs from being filled. The result is to help develop new approaches for recruitment.

\section{MATERIALS AND METHODS}

\section{Participants}

All residents who were enrolled in a medical genetics residency or combined (pediatric, internal medicine, or maternal fetal medicine) medical genetics residency and those who wrote the 2011 medical genetics in-service examination were invited to participate. Members of the Program Directors Association (PDA), a special interest group (SIG) of the Association of Professors of Human and Medical Genetics (APHMG), were also invited to complete a separate online survey.

\section{Procedure}

After obtaining institutional review board approval from the Human Investigation Committee at Wayne State University (WSU), the chairperson of the PDA sent an e-mail invitation to the 127 residents who wrote the 2011 genetics in-service examination. ${ }^{5}$ The e-mail contained a SurveyMonkey.com link, which led to a consent and survey. Laboratory trainees also received the e-mail; however, only data from MD/DO medical genetics residents were used for this study. Respondents were allowed to skip questions.

As a quality improvement project, medical genetics program directors who were members of the PDA were sent an e-mail invitation from the principal investigator through the PDA listserv. The e-mail contained a separate SurveyMonkey.com link, which led to the program directors' survey.

All completed surveys were accessible only to the principal investigators at WSU.

\section{Surveys}

The resident survey was a modified version of the "National Survey of Factors Influencing Career Choice" created by the Clerkship Directors in Internal Medicine (CDIM) Task Force on Enhancing Student Interest in Internal Medicine Careers ${ }^{11}$ and included questions regarding demographic information and their exposure to the field before entering it. The survey was designed such that the questions emphasized medical school as the time when career decisions are made, although the majority of trainees are in a categorical program requiring previous residency training. The reason for this was to determine whether we are capturing the attention of medical students who are eligible to enter a combined residency program or whether we can retain their interest as they progress through prerequisite residency training to medical genetics training (see Supplementary Table S1 online).

The program director's survey queried program directors about their current recruitment efforts and institutional financial support (see Supplementary Table S2 online).

\section{Data analysis}

Descriptive statistics were used to report the findings of both surveys.

\section{RESULTS}

\section{Resident survey}

Seventy-seven individuals stating to be clinical medical genetics trainees completed the resident survey. Four were removed from the analysis because they did not enter what type of program they were currently enrolled in, which reduced the likelihood that the respondent was a clinical medical genetics trainee. This represented a 57\% participation rate.

Demographics. Of the 73 respondents, $51(70 \%)$ were in a categorical medical genetics residency, $16(22 \%)$ in pediatricsmedical genetics, $4(5 \%)$ in maternal fetal medicine-medical genetics, and $2(3 \%)$ in internal medicine-medical genetics. Previous training included pediatrics $(\mathrm{n}=33)$, internal medicine $(\mathrm{n}=7)$, obstetrics and gynecology $(\mathrm{n}=11)$, family practice $(\mathrm{n}=$ 5 ), and others $(n=3)$. The age ranged from 26 to 50 years (mean: 34 years), with two individuals older than 50 years. Sixty-five percent were women. Sixty-nine percent classified themselves as white, $73 \%$ were married or had a domestic partner, and 57\% had children. Furthermore, 43 (59\%) respondents had an MD degree, whereas an additional 21 (29\%) respondents had both an MD and an additional degree. Fifty-seven percent attended medical school in the United States. Twenty-seven (42\%) participants reported no debt, with the remaining participants reporting debt ranging from $\$ 10,000-\$ 350,000$, (median $=$ $\$ 120,000)$.

Factors influencing decision making. Forty-six (63\%) residents chose to enter a medical genetics residency during their initial residency program or a subsequent subspecialty fellowship program. Twenty-three $(32 \%)$ did not take a medical genetics elective before entering the medical genetics residency. The majority of those who did take a medical genetics elective stated it was influential in their decision to enter medical genetics. Six respondents (8\%) stated that they had a medical genetics SIG at their medical school. Of those six, one attended the corresponding SIG and reported that it influenced the decision to enter a medical genetics residency. The top three factors that influenced their decision to enter a medical genetics residency were as follows: (1) having a geneticist as a mentor (50\%), (2) previous research in genetics 
(35\%), and (3) a medical school genetics course (33\%). When asked what one thing influenced them the most to enter a genetics residency, they listed "mentor." Nine individuals (13\%) either strongly agreed or agreed with the statement, "My medical school experience provided me with enough insight into what a medical geneticist does to make an informed decision about medical genetics as a career." Thirty (43\%) residents first learned that medical genetics was a primary specialty during medical school. A slightly higher percentage of international graduates learned of medical genetics during their initial residency-35\% vs. $23 \%$ of US graduates. More US graduates learned about medical genetics as a career in high school or undergraduate studies. When asked why they chose a career in medical genetics, the top three factors listed were as follows: (1) "the intellectual challenge of genetics," (2) "ongoing advancements in the field," and (3) "my feelings of taking care of a variety of patients." The top three negative factors listed were (1) "paperwork and charting," (2) "the need to bring work home," and (3) "the loans I have to repay."

\section{Program directors' survey}

Thirty-five program directors completed the survey ( $70 \%$ of the 50 accredited programs in 2011).

Of the program directors surveyed, $57 \%$ reported that their programs filled with at least one resident per year. For the programs that did not fill at least one position each academic year, the following reasons were provided: (1) no qualified applicants (40\%), (2) interviewed qualified applicants who matched elsewhere (40\%), and (3) no funding (13\%). For the 2011-2012 academic year specifically, $69 \%$ of the program directors who responded to the survey filled a position. Fifty-two percent of those who filled positions in 2011-2012 did so through either a categorical or combined match. (This was before the National Residency Matching Program's all-in Match policy, and we would expect that the percentage of trainees entering through the Match will increase.)

Recruiting methods. Program directors stated that the most common opportunities their institutions offered to medical students included electives (89\%), clinical shadowing opportunities (64\%), unpaid research opportunities (53\%), paid research opportunities (39\%), unpaid summer externship (31\%), paid summer externship (28\%), medical genetics SIG (25\%), and journal club (17\%).

Eighty-five percent of programs encourage residents from other specialties to consider completing a medical genetics residency after their initial residency training. Opportunities offered to those residents included research (64\%), mentoring (50\%), a formal invitation to attend medical genetics grand rounds (29\%), and a formal invitation to journal club (14\%). The types of electives offered to residents included pediatric genetics (92\%), reproductive genetics (35\%), adult genetics $(28 \%)$, combined or tailored genetics $(24 \%)$, and others (i.e., neurogenetics, dentistry, pathology, laboratory, metabolics: $17 \%)$.
Social media was used by $6 \%$ of programs, with the success of such recruiting strategies being ranked "neutral" or "not very successful" (100\%). Seventy-five percent of programs have a website, with $19 \%$ stating this approach has been successful in recruiting and $60 \%$ ranking it as neutral or not very successful. Sixty-one percent of programs use their current residents to recruit individuals to the field. When queried, "What was your most successful recruiting method?" $80 \%$ of program directors responded with, "direct contact with residents or medical school students" or "word of mouth."

\section{DISCUSSION}

The information collected from both surveys revealed valuable information regarding recruitment efforts in medical genetics. Of critical importance, geneticists need to develop contact with medical students/residents early in their medical training, providing positive exposure in all areas of the field. There are several key concepts that may help target and support recruitment, providing the field with the opportunity to develop new recruitment strategies.

The "typical" medical genetics resident who answered the survey was a 34-year-old white married woman with children, enrolled in a 2-year program, and who had previous pediatric residency training. She earned her MD degree in the United States, leaving her with a median debt of $\$ 120,000$. This debt is similar to the national statistics of $\$ 120,000-\$ 140,000$ provided by the American Association of Medical Colleges for medical school graduates between 2006 and 2009. ${ }^{12}$ The field of medical genetics appears to capture the attention of women, with $65 \%$ in our study being female respondents. This percentage is higher than the percentage (54\%) of female categorical medical genetics residents reported by the Accreditation Council for Graduate Medical Education during the same academic year. ${ }^{13}$ However, data were not available for combined trainees, and $20 \%$ of our respondents were in combined programs. Furthermore, this is lower than the percentage of women who graduated from pediatric residency programs and took the American Board of Pediatric Examination in $2012(71 \%)^{14}$ but higher than the percentage of female graduates of medical school in the year $2011(48 \%) .{ }^{15}$ It is imperative that advertising materials be gender neutral in presentation. Furthermore, program incentives should take into consideration child-care issues because the majority of women and men entering the field have children.

Another interesting demographic is the number of research scientists in residency programs. Nineteen percent of respondents hold both an MD and a PhD (Doctor of Philosophy), of whom $69 \%$ were US graduates. In addition, $75 \%$ of the respondents stated that genetic research opportunities somewhat positively or positively influenced their career choice. The 2-year medical genetics residency and the maternal fetal medicine-medical genetics combined program have 6 months of available elective time, which can be used for research. The 5 -year combined pediatrics-medical genetics and combined internal medicine-medical genetics programs provide 12 months of elective time, although this will be decreased to 6 
months for the pediatrics-medical genetics program in 2014 . It will be important to monitor whether this makes a difference in the quantity or quality of combined residency trainees. The available research time supports those candidates who desire research experience and may be the reason why there are so many residents with dual degrees. On the contrary, this statistic may be a hindrance to recruitment because potential trainees may inaccurately have the impression that they need a $\mathrm{PhD}$ to be competitive in the Match. Geneticists should keep this in mind when discussing the opportunities that various residency programs offer to students or residents.

There are mixed reports regarding the notion that the increased debt that medical students incur influences them to choose higher-paying specialties. ${ }^{16-18}$ Initially, it would appear that our survey findings could support that career choice is influenced by debt, with $42 \%$ of residents having no debt. In addition, residents ranked "the amount of loan debt to repay" as the third highest negative influence in choosing medical genetics. However, our survey respondents who graduated from a US medical school revealed that $46 \%$ had debts greater than $\$ 120,000$, which is similar to that of respondents in other primary specialties (e.g., pediatrics, $45 \%){ }^{11}$ This suggests that US medical school students' choice to enter medical genetics with higher debt is no different than the same for other primary medical specialties.

Only nine individuals (13\%) either strongly agreed or agreed with the statement, "My medical school experience provided me with enough insight into what a medical geneticist does to make an informed decision about medical genetics as a career." Sixty-seven percent of US graduates stated that they disagreed or strongly disagreed with this statement. These statistics are surprising because $43 \%$ of residents stated they had learned about medical genetics being a primary medical specialty during medical school. Hence, although students are hearing about the specialty, they are not provided enough exposure to grasp the nuances of the career. To incorporate clinical applications of genomic medicine, it is important to ensure that clinical geneticists, and not solely basic scientists, are teaching genetics to medical students, especially during the course typically offered during the first or second year of medical school. Moreover, incorporating medical genetics into the third and fourth clinical years may provide more insight into and enthusiasm for medical genetics as a career. Some schools already have an integrated genetics curriculum. It would be beneficial to determine whether more students enter genetics from schools with this approach versus the more traditional route of a single genetics course and whether the involvement of clinical geneticists affects these numbers.

The majority of US medical graduates who completed an elective in genetics before entering a medical genetics residency stated that it strongly influenced them to enter the field. Eighty-nine percent of programs surveyed offered medical genetics electives to medical students. Although it appears that electives are available, $23 \%$ of US graduates did not take electives. When the data are broken down by those who took an elective and those who did not, there was a difference based on which medical genetics track was chosen. Eighty-six percent of residents surveyed who took a medical genetics elective during medical school had previous pediatrics training or entered a combined pediatrics-medical genetics program directly from medical school. The majority (67\%) of those who did not take an elective had previous training in obstetrics and gynecology and half of them entered a combined maternal fetal medicinemedical genetics training program. The program directors' survey showed that only $35 \%$ of programs offered reproductive genetics electives. This might suggest a missed opportunity-if more institutions offered an elective in reproductive genetics, perhaps we could attract additional trainees with an interest in that area. If a reproductive genetics elective is not available at one's institution, the genetics faculty could determine whether a local facility would provide such an elective and promote that option to those interested in obstetrics and gynecology.

"Mentor" was the primary factor influencing trainees to enter medical genetics. This is a key component in recruiting residents, and the program directors' survey supports mentorship as the foremost recruiting tool. As residents and medical students rotate through genetics clinics, it is important that a geneticist takes the time to nurture those choosing the rotation, keeping in mind that each student or resident choosing a genetics elective is a potential future medical genetics resident. Mentoring could include inviting prospective trainees to grand rounds, journal club, or other genetics-related activities to help immerse them into the field.

When looking at program recruitment efforts, it is striking that only $75 \%$ of programs have a website. In addition, social media is used by only $6 \%$ of programs. Social media use among students in the medical professions is reported to be $\sim 80 \% .^{19}$ Although no published data were found in a PubMed search regarding the use of social media for residency recruitment, it is logical to assume that it is important to use the resources that current students use in their everyday lives to at least introduce, captivate, and possibly recruit students into genetics. The nonprofit organization Student Doctor Network, http://studentdoctor.net, is mentioned in the literature as a site frequently used by medical students for career guidance. ${ }^{20}$ This may be a site to initiate conversation with medical students regarding genetics. At a minimum, genetics programs should have a website to help in showcasing their institutions' genetics programs to potential trainees.

SIGs were available at some institutions, yet were noted as not being helpful in recruitment. Although it is difficult to assess the quality of such programs because the SIGs are primarily student driven with faculty input, the number of SIGs is increasing, and this will hopefully lead to an increased number of students interested in this career path. It was significant that of seven residents who stated there was a medical genetics SIG at their medical school, only one attended the group. Advertising the SIG during the introductory medical genetics course may be helpful in increasing the awareness of the SIG at that institution. In 2011, the American College of Medical Genetics and 
Genomics (ACMG) initiated a medical genetics SIG affiliation program with medical schools. Currently, there are only 13 medical schools listed on the ACMG website. ${ }^{21}$ "Membership provides groups access to unique educational resources, opportunities for networking, and information about careers in medical genetics."22 Further research should be conducted to determine whether the support of ACMG increases the participation in such a group and whether it influences career choice.

These survey results were initially presented at the 2011 annual meeting of the APHMG. One recommendation made at that time was to create a task force composed of individuals representing the various organizations involved in genetics education and training. Thus, the ACMG created the Task Force in Medical Genetics Education and Training. The task force is organized through the ACMG and includes representatives from ACMG, APHMG, American Society of Human Genetics, PDA, and the ACMG Foundation. Another recommendation presented at the 2011 APHMG meeting was to develop a video devoted to a career in medical genetics specifically aimed at future trainees. Through the support of the ACMG and the ACMG Foundation, an excellent video was produced and can be found at TheACMGChannel of YouTube, which can be used by residency programs with an option to personalize it to fit the specific residency program's needs. ${ }^{23}$

Another resource for geneticists to help introduce students to the field is through the student section on the ACMG website. This site includes publications, presentations regarding a career in medical genetics, and information on the ACMG Summer Genetics Scholars Program. In addition, ACMG offers student membership discounts to join the ACMG and special opportunities for medical students at their annual meeting. These programs are being closely monitored to determine whether they have a positive impact on increasing the number of individuals entering the field.

There are several limitations to consider regarding these survey results, in addition to implications for future research. To begin with, the resident survey was not sent to residents who chose other fields. It would be important to know reasons why others do not choose medical genetics. Moreover, because there are many different career paths for the medical geneticist, it was difficult to separate out each subgroup of trainees and program directors of those subgroups. However, for the purpose of these surveys, it was important to obtain aggregate data that would be useful when analyzed as a whole. In fact, some might consider that the multiple training tracks are a liability of the specialty itself. We recognize that the timings of the trainees' decision to enter a residency, the training schedules, and the lengths of training are very different; perhaps a future survey can investigate those differences more closely. More data also need to be obtained from program directors, such as whether program directors prefer training residents with previous residency training or those entering a combined program directly from medical school; there are certainly benefits and limitations of both tracks. Furthermore, additional data need to be collected to better understand what specific genetic opportunities exist at each institution and whether successes at some programs can be used by other programs (e.g., analyzing the number of contact hours with trainees during the introductory medical genetics course, the clinical years, and other settings). Finally, one needs to take into consideration the likelihood that responses of the program director relate to the success of their individual programs (e.g., how many residents are currently enrolled in their program and their success rate in filling their available positions). If gathered, the additional information from both residents and program directors would only enhance recruitment strategies.

In summary, geneticists should become more proactive in providing resources to students to help them understand what a career as a medical geneticist is and initiate a mentoring relationship early in medical training with those students/residents who show true interest in the field. Mentorship should begin early and continue throughout training with frequent positive interactions. Several suggestions to help with engaging medical students in the field include the following: (i) provide gender-neutral advertisements; (ii) consider the research component of the residency as a positive aspect for those with research interest, yet be mindful that it could hinder those not interested in research; (iii) become involved in the introductory medical genetics course and use that as an opportunity to introduce medical genetics as a primary medical specialty; (iv) create a useful website; (v) become involved in or help create a medical genetics SIG; and (vi) use currently available resources, such as the ACMG website and recruitment video. For the genetics profession in general, future research should be conducted to determine the effectiveness of any implemented programs. Although there is no expectation that the specialty of medical genetics will expand at a breakneck pace, without such initiatives, it will be a challenge for geneticists to fulfill all the areas of medicine that they are being called upon to provide expertise in.

\section{SUPPLEMENTARY MATERIAL}

Supplementary material is linked to the online version of the paper at http://www.nature.com/gim

\section{DISCLOSURE}

The authors declare no conflict of interest.

\section{REFERENCES}

1. Cooksey JA, Forte G, Benkendorf J, Blitzer MG. The state of the medical geneticist workforce: findings of the 2003 survey of American Board of Medical Genetics certified geneticists. Genet Med 2005;7:439-443.

2. Scheuner MT, Sieverding P, Shekelle PG. Delivery of genomic medicine for common chronic adult diseases: a systematic review. JAMA 2008;299:13201334.

3. Battista RN, Blancquaert I, Laberge AM, van Schendel N, Leduc N. Genetics in health care: an overview of current and emerging models. Public Health Genomics 2012;15:34-45

4. Number of Certified Specialists in Genetics. American Board of Medical Genetics, 2011. http://www.abmg.org/pages/resources_certspecial.shtml. Accessed 10 January 2013

5. Robin NH, Reid Sutton V, Caldwell J, Jackson J, Irons M, Demmer L; Members of in-service exam subcommittees. The development and implementation 
of an in-service exam for medical genetics residency programs. Genet Med 2012;14:552-557.

6. ACGME Data Resource Book, Academic Year 2011-2012. Department of Applications and Data Analysis, ACGME: Chicago, IL, 2012.

7. National Resident Matching Program, Results and Data: Specialties Matching Service $^{\text {SM }} 2012$ Appointment Year. National Resident Matching Program: Washington, DC, 2012.

8. National Resident Matching Program, Results and Data: Specialties Matching Service $^{\text {SM }} 2013$ Appointment Year. National Resident Matching Program: Washington, DC, 2013.

9. National Resident Matching Program, Results and Data: 2013 Main Residency Match ${ }^{\mathrm{SM}}$. National Resident Matching Program: Washington, DC, 2013.

10. Korf BR, Feldman G, Wiesner GL. Report of Banbury Summit meeting on training of physicians in medical genetics, October 20-22, 2004. Genet Med 2005;7:433-438.

11. Hauer KE, Durning SJ, Kernan WN, et al. Factors associated with medical students' career choices regarding internal medicine. JAMA 2008;300:11541164.

12. 2009 GQ Medical School Graduation Questionnaire: All Schools Summary Report FINAL. Academic Affairs, Association of American Medical Colleges: Washington, DC, 2009.

13. ACGME Data Resource Book, Academic Year 2010-2011. Department of Applications and Data Analysis, ACGME: Chicago, IL, 2011.

14. The American Board of Pediatrics 2012 Workforce Data. American Board of Pediatrics. http://www.abp.org. Accessed 11 July 2013.
15. Castillo-Page L. Diversity in Medical Education Facts and Figures, 2012. Association of American Medical Colleges, Diversity Policy and Programs: Washington, DC. Fall 2012.

16. Palmeri M, Pipas C, Wadsworth E, Zubkoff M. Economic impact of a primary care career: a harsh reality for medical students and the nation. Acad Med 2010;85:1692-1697.

17. Kahn MJ, Markert RJ, Lopez FA, Specter S, Randall H, Krane NK. Is medical student choice of a primary care residency influenced by debt? MedGenMed 2006;8:18.

18. Grayson MS, Newton DA, Thompson LF. Payback time: the associations of debt and income with medical student career choice. Med Educ 2012:46:983-991.

19. Giordano C, Giordano C. Health professions students' use of social media. J Allied Health 2011;40:78-81.

20. von Muhlen M, Ohno-Machado L. Reviewing social media use by clinicians. J Am Med Inform Assoc 2012;19:777-781.

21. ACMG Student Interest Group Program. ACMG: Translating Genes into Health(C. Accessed 20 February 2013. https://www.acmg.net/ACMG/ STUDENTS/StudentInterestGroups/ACMG/About ACMG/ACMG_Student_ Groups.aspx?hkey=75491646-ea2b-4140-9d4c-b540701a82a5

22. ACMG Student Interest Group Program. ACMG: Translating Genes into Health@. Accessed 20 February 2013. https://www.acmg.net/ACMG/ STUDENTS/ACMG/ACMG_Student_Interest_Groups.aspx?hkey=3035864a587a-4194-ae8c-7f5f5ed5c569

23. "Medical Genetics is the Future of Medicine" Oct. 2012, TheACMGChannel. YouTube. http://www.youtube.com/theacmgchannel. Accessed 20 February 2013 